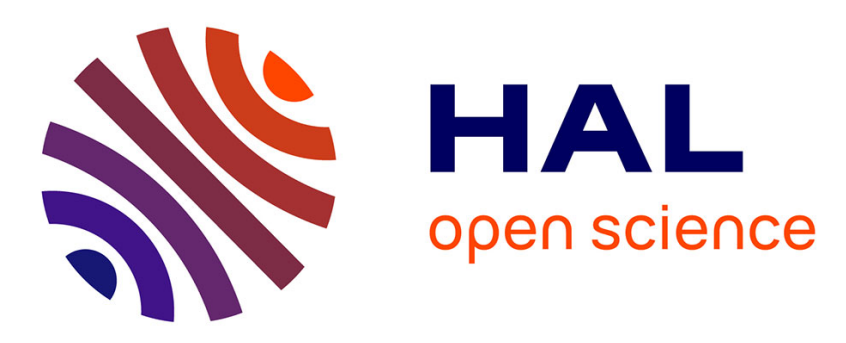

\title{
Directed lattice animals in 2 dimensions : numerical and exact results
}

\author{
J.P. Nadal, B. Derrida, J. Vannimenus
}

\section{To cite this version:}

J.P. Nadal, B. Derrida, J. Vannimenus. Directed lattice animals in 2 dimensions : numerical and exact results. Journal de Physique, 1982, 43 (11), pp.1561-1574. 10.1051/jphys:0198200430110156100 . jpa-00209536

\section{HAL Id: jpa-00209536 https://hal.science/jpa-00209536}

Submitted on 1 Jan 1982

HAL is a multi-disciplinary open access archive for the deposit and dissemination of scientific research documents, whether they are published or not. The documents may come from teaching and research institutions in France or abroad, or from public or private research centers.
L'archive ouverte pluridisciplinaire HAL, est destinée au dépôt et à la diffusion de documents scientifiques de niveau recherche, publiés ou non, émanant des établissements d'enseignement et de recherche français ou étrangers, des laboratoires publics ou privés. 


\title{
LE JOURNAL DE PHYSIQUE
}

Classification

Physics Abstracts

$05.20-05.50$

\section{Directed lattice animals in 2 dimensions : numerical and exact results}

\author{
J. P. Nadal (*), B. Derrida and J. Vannimenus (*) \\ CEN-Saclay, Division de la Physique, Service de Physique Théorique, 91191 Gif-sur-Yvette cedex, France \\ (*) Groupe de Physique des Solides, ENS, 24, rue Lhomond, 75231 Paris cedex 5, France
}

(Reçu le 28 mai 1982, accepté le 21 juillet 1982)

\begin{abstract}
Résumé. - Nous étudions divers modèles d'animaux dirigés (polymères branchés) sur un réseau carré. Nous présentons une méthode de matrice de transfert pour calculer les propriétés de ces animaux dirigés quand le réseau est un ruban de largeur finie. En utilisant la renormalisation phénoménologique, nous obtenons des prédictions précises pour les exposants qui décrivent la longueur et la largeur moyennes de grands animaux $\left(v_{\|}=9 / 11\right.$ et $\left.v_{\perp}=1 / 2\right)$. Pour un modèle particulier d'animaux de sites nous présentons et prouvons certains résultats exacts (découverts numériquement), sur la constante de connectivité et le vecteur propre de la matrice de transfert pour une valeur propre égale à 1 . Nous proposons enfin une conjecture pour le nombre d'animaux, qui généralise l'expression devinée par Dhar, Phani et Barma.
\end{abstract}

\begin{abstract}
We study several models of directed animals (branched polymers) on a square lattice. We present a transfer matrix method for calculating the properties of these directed animals when the lattice is a strip of finite width. Using the phenomenological renormalization, we obtain accurate predictions for the connective constants and for the exponents describing the length and the width of large animals $\left(v_{\|}=9 / 11\right.$ and $\left.v_{\perp}=1 / 2\right)$. For a particular model of site animals, we present and prove some exact results that we discovered numerically concerning the connective constant and the eigenvector of the transfer matrix when the eigenvalue is one. We also propose a conjecture for the number of animals which generalizes the expression guessed by Dhar, Phani and Barma.
\end{abstract}

1. Introduction. - Among the many generalizations of the classical percolation problem, the introduction of a preferred direction for the available bonds has the remarkable property that it changes the universality class of the model considered $[1,2]$. This is one of the reasons for the recent wave of interest in directed percolation [3-8] and it seems worthwhile to investigate the effects of directionality on other systems.

The first model one can think about is the problem of fully directed polymers, i.e. walks for which each step has a positive projection on the preferred direction. Such a walk can obviously be decomposed into a random walk perpendicular to the direction and a forward walk parallel to the direction. Due to this simplification, the problem becomes trivial, even for self-avoiding walks because all directed polymers satisfy the excluded volume constraint.

The next model is the problem of directed lattice animals (or branched polymers). One wants to study the statistical properties of connected clusters obeying the following rule : there exists a particular site (the root) from which all the other sites in the cluster can be reached via a path that never goes opposite to the preferred direction. This problem was attacked recently by two methods : Day and Lubensky [9] developed a field theory approach of the problem. They found that the upper critical dimensionality was 7 and they calculated the critical exponents to first order in $\varepsilon(\varepsilon=7-d)$. On the other hand 
Redner and Yang [10] and Dhar, Phani and Barma [11] studied the problem on lattices by performing direct enumerations up to a maximum size. This maximum size is significantly higher than the one which can be reached for non-directed lattice animals. The reason is that the directional constraint greatly simplifies the problems, at least in numerical studies. The same simplification can also be observed for percolation. By the enumeration method, they estimated the asymptotic number of animals (in the large size limit) and the critical exponents. Moreover, Dhar, Phani and Barma [11] conjectured some exact expressions for the number of directed animals in dimension 2.

In the present paper, we study the problem of directed lattice animals in two dimensions using transfer matrices. As in the case of non-directed animals [12], the transfer matrix method allows animals on strips of finite width to be studied. But contrary to the enumeration method where the animals are constructed up to a maximum size, the transfer matrix method does not limit the size of animals. It only limits the width of the strips where the animals are drawn. Once the transfer matrices were written, we could apply the phenomenological renormalization $[13,15,12]$ to estimate the exponents in dimension 2. We investigated several cases (with sites, with bonds, without loops, partially directed) and we found that the universality class does not seem to depend on the details of the model. Our numerical results indicate that the exponents $v_{\|}$ and $v_{\perp}$ (to be defined in section 2) are very well approximated by $v_{\|}=9 / 11$ and $v_{\perp}=1 / 2$.

In the last section, we study in more details one particular model of site animals for which we could prove some exact results. We show that the connective constant, $\mu$, is 3 for this model and that the eigenvector of the transfer matrix has a simple form in some cases. We also propose (without proof) a generalization of the conjecture of Dhar, Phani and Barma [11] for the expression of the number of lattice animals of $s$ sites. If proved correct this conjecture would give the exact value of the two exponents $\theta$ and $v_{\perp}$. However the last exponent of interest $v_{\|}$ seems to be much more difficult to obtain.

2. Definition of the models. - We present in this paper results concerning four different models of directed animals on the square lattice. For all these models, there is a preferred direction on the lattice. The only allowed configurations of the animals are such that any element of the cluster can be reached from the root by a path which never goes opposite to that direction (Fig. 1).

- In model A (Fig. 1a) the animals are clusters of $s$ sites and the preferred direction lies along the diagonal of the square lattice.

- Model B (Fig. 1b) differs only from model A by the fact that the configurations with loops are for-

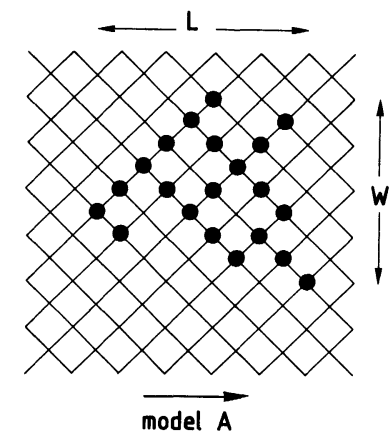

la

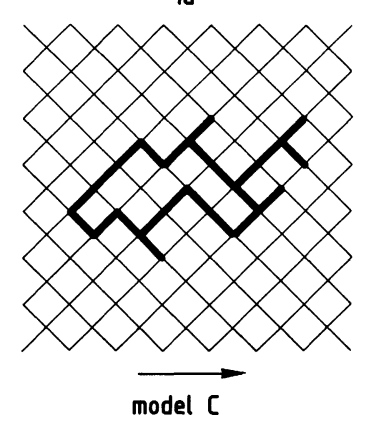

1c

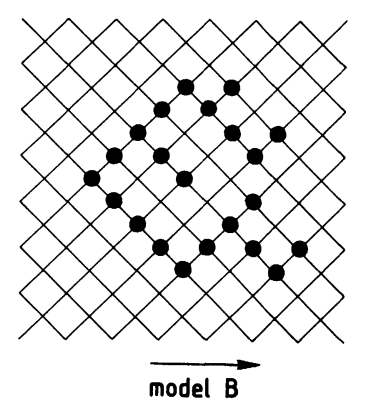

ib

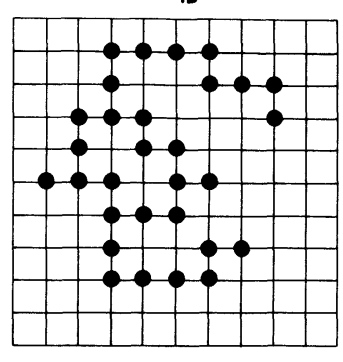

model $D$

id

Fig. 1. - Typical clusters of the four models A, B, C and D studied in the present paper. The leftmost site is the root of the cluster. The arrows indicate the preferred direction.

bidden. This means that each site of the cluster can be reached from the root by one and only one path. The animals have the topology of trees.

- In model C (Fig. 1c), the elements of the cluster are bonds and the preferred direction lies again along the diagonal of the square lattice.

- In model D (Fig. 1d), the elements are sites, the loops are allowed and the direction is parallel to the horizontal lines of the lattice. One can say that the animals are partially directed since the vertical lines are no longer oriented.

By analogy with the problem of polymers [14] and of non-directed lattice animals [15], the basic quantities one wants to study are : the number $\Omega_{s}$ of different configurations of directed animals composed of $s$ elements and the average size of these animals. For directed animals, one needs to introduce two average sizes (Fig. 1a) :

- The average length $L$ which is the average distance between the two most remote elements along the preferred direction.

- The average width $W$ which is the average distance between the two most remote elements in the direction perpendicular to the preferred direction.

For $s$ sufficiently large, one expects $\Omega_{s}, L$ and $W$ to have simple asymptotic forms :

$$
\begin{aligned}
\Omega_{s} & \sim \mu^{s} s^{-\theta} \\
L & \sim s^{v_{\|}} \\
W & \sim s^{v_{\perp}} .
\end{aligned}
$$


The growth parameter $\mu$ is called the connective constant and depends on the model considered, but the exponents $\theta, v_{\|}$and $v_{\perp}$ should be universal and depend only on the dimensionality. Physically one expects $v_{\|}$to be larger than $v_{\perp}$ because the animals have more freedom to grow in the preferred direction.

The four models presented above were chosen to see if they belonged to the same universality class and if the details of the model (such as the presence of loops, the relative position of the preferred direction and of the lattice, the nature of the elements of the animals) were irrelevant.

3. Transfer matrix. - The transfer matrix is a very useful tool for calculating the properties of a number of problems in statistical mechanics. It is well adapted to the study of lattices which are infinite in one direction and finite in the other directions : strips of finite width, bars of finite section... In the present work, we have used this transfer matrix method to study the problem of directed animals. For the four models A, B, C and D, we have chosen the strips to be infinite along the preferred direction and to have periodic boundary conditions in the perpendicular direction. Strips with periodic boundary conditions are easy to realize even when the preferred direction is along the diagonal of the lattice [5] (see appendix A). We explain here how one can write the transfer matrix for model A. The same method can be applied to the other models without any difficulty.

Following Derrida and de Seze [12], we first define a generating function $G_{0 \mathbf{R}}$ for the total number $\Omega_{s}(0, \mathbf{R})$ of directed animals with $s$ sites and which contain both sites 0 and $\mathbf{R}$ on the lattice

$$
G_{0 \mathbf{R}}=\sum_{s} x^{s} \Omega_{s}(0, R) .
$$

Sites 0 and $\mathbf{R}$ may be anywhere in the animal.

When the two points 0 and $\mathbf{R}$ are chosen far away along the strip, the function $G_{0 \mathbf{R}}$ behaves like

$$
G_{0 \mathbf{R}} \sim[\lambda(x)]^{R} .
$$

We are now going to justify the asymptotic behaviour (5) and to show that $\lambda(x)$ is the largest eigenvalue of the transfer matrix.

The transfer matrix is very easy to write for this problem. Consider a strip of width $n$. Let us define $2^{n}-1$ quantities $\psi_{R}(\mathcal{C}, x)$ :

$$
\psi_{R}(\mathcal{C}, x)=\sum_{s} x^{s} \omega_{s}(\mathcal{C}, R)
$$

where $\omega_{s}(\mathcal{C}, R)$ is the number of lattice animals with a root $\mathcal{C}$ at column 0 and containing one or several sites at column $R$. C represents a given set of occupied sites at column 0 and any animal which is counted in $\omega_{s}(\mathcal{C}, R)$ is a cluster of $s$ sites with the following property : any site of the cluster can be reached from at least one site of the root by a path which never goes opposite to the preferred direction. Obviously the number of configurations $\mathcal{C}$ (i.e. the number of possible roots) is $2^{n}-1$ because the section of the strip has $n$ lattice sites and each lattice site of this section may be occupied or empty (of course the root with 0 occupied site plays no role).

Once the $\psi_{R}(\mathcal{C}, x)$ have been defined, one can easily write recursion relations :

$$
\psi_{R+1}(\mathcal{C}, x)=x^{m(\mathbb{C})} \sum_{\mathbb{C}^{\prime}}^{\prime} \psi_{R}\left(\mathbb{C}^{\prime}, x\right)
$$

where $m(\mathcal{C})$ is the number of occupied sites in the root $\mathcal{C}$.

This recursion relation can be understood by noticing that an animal of length $R+1$ with a root $\mathcal{C}$ can always be composed of its root $\mathcal{C}$ which occupies 1 column and of an animal of length $R$ with a root $\mathrm{C}^{\prime}$.

The sum $\sum^{\prime}$ over the configurations $\mathfrak{C}^{\prime}$ is restricted to the successors of $\mathcal{C}$, i.e. configurations $\mathrm{C}^{\prime}$ which are allowed to follow configuration $\mathcal{C}$. This means that if we have configuration $\mathcal{C}$ at column 0 and configuration $\mathfrak{C}^{\prime}$ at column 1 , any site of configuration $\mathbf{C}^{\prime}$ is connected to at least one site of configuration $\mathrm{C}$. The recursion (7) has obviously a matrix form. It can be written as :

$$
\psi_{R+1}(\mathcal{C}, x)=x^{m(\mathcal{C})} \sum_{\mathrm{e}^{\prime}} M\left(\mathcal{C}, \mathcal{C}^{\prime}\right) \psi_{R}\left(\mathrm{C}^{\prime}, x\right)
$$

or

$$
\psi_{R+1}(\mathcal{C}, x)=\sum_{\mathcal{C}^{\prime}} T\left(\mathcal{C}, \mathbb{C}^{\prime}\right) \psi_{R}\left(\mathcal{C}^{\prime}, x\right)
$$

where

$$
M\left(\mathcal{C}, \mathcal{C}^{\prime}\right)=1 \text { if } \mathcal{C}^{\prime} \text { is a successor of } \mathcal{C}
$$

and

$$
M\left(\mathcal{C}, \mathrm{C}^{\prime}\right)=0 \quad \text { otherwise }
$$

and

$$
T\left(\mathcal{C}, \mathcal{C}^{\prime}\right)=x^{m(\mathcal{C})} M\left(\mathcal{C}, \mathcal{C}^{\prime}\right)
$$

The matrix $T$ is the transfer matrix. The relation (8) justifies the asymptotic behaviour of $G_{0 \mathbf{R}}(x)$ given in equation (5) and allows $\lambda(x)$ to be calculated as the largest eigenvalue of matrix $T$. The choice of periodic boundary conditions reduces the size of the transfer matrix when one takes advantage of the translational symmetry around the strip. For example, this size is reduced to 5 instead of 15 for a strip of width 4 (see appendix A).

If we want to enumerate the directed animals on a strip, we can also use the matrix $M$. Let us call $\Omega_{s}(\mathcal{C})$ the number of directed animals of $s$ sites with a root $\mathrm{C}$. One has :

$$
\Omega_{s^{\prime}}(\mathcal{C})=\delta_{s, 0}+\sum_{\mathcal{C}^{\prime}} M\left(\mathcal{C}, \mathbb{C}^{\prime}\right) \Omega_{s}\left(\mathcal{C}^{\prime}\right)
$$

with $s^{\prime}=s+m(\mathcal{C})$.

If we use the fact that

$$
\Omega_{s}(\mathcal{C})=0 \quad \text { if } \quad s<m(\mathcal{C})
$$


and

$$
\Omega_{m(\mathrm{C})}(\mathrm{C})=1,
$$

the recursion relation (12) allows us to calculate the $\Omega_{s}(\mathcal{C})$ without upper limit for $s$.

Defining now the generating functions $H(\mathrm{C}, x)$ of the $\Omega_{s}(\mathcal{C})$ by :

$$
H(\mathcal{C}, x)=\sum_{s} x^{s} \Omega_{s}(\mathcal{C}) .
$$

One deduces from equations (12) and (13) that the functions $H(\mathrm{C}, x)$ are solutions of the following set of linear equations.

$$
H(\mathcal{C}, x)=x^{m(\mathcal{C})}\left(1+\sum_{\mathcal{C}^{\prime}} M\left(\mathcal{C}, \mathcal{C}^{\prime}\right) H\left(\mathcal{C}^{\prime}, x\right)\right) .
$$

For strips of finite width, the matrix $M$ is finite. Therefore the eigenvalue $\lambda(x)$ and the generating functions $H(\mathrm{C}, x)$ can be calculated for any value of $x$. The only limitation comes from the fact that the size of the matrix increases exponentially with the width $n$ of the strip.

4. Results on strips of finite width - The largest eigenvalue $\lambda_{n}(x)$ of the transfer matrix corresponding to a strip of width $n$ can be calculated either analytically for narrow strips, or numerically but with a very high accuracy for larger strips. As an example, we give the expression of $\lambda_{n}(x)$ for strips of width $n=2,3$ and 4 in the case of model A :

$$
\lambda_{2}(x)=2 x+x^{2}
$$

$\lambda_{3}(x)$ is the largest root of

$$
\lambda^{2}-\lambda\left(2 x+3 x^{2}+x^{3}\right)+3 x^{3}+2 x^{4}=0
$$

$\lambda_{4}(x)$ is the largest root of :

$$
\begin{aligned}
& \lambda^{3}-\lambda^{2}\left(x^{4}+4 x^{3}+4 x^{2}+2 x\right)+ \\
& \quad+\lambda\left(2 x^{6}+6 x^{5}+8 x^{4}+5 x^{3}\right)-x^{7}+2 x^{5}=0
\end{aligned}
$$

(see appendix A).

As strips are lattices which are infinite in one direction, one can study the large $s$ behaviour of the number of animals on these strips. This leads to define the connective constant $\mu_{n}$ of the model on a strip of width $n$.

For site lattice animals, the number of different animals of $s$ sites on a strip of width $n$ and with a root $\mathcal{C}$ behaves by definition of $\mu_{n}$ as :

$$
\Omega_{s}(\mathcal{C}) \sim\left(\mu_{n}\right)^{s} a(\mathcal{C}) .
$$

One should notice that in (19), it is only the prefactor $a(\mathcal{C})$ which depends on the form of the root $\mathcal{C}$. The connective constant $\mu_{n}$ is the same for all the roots. This is a consequence of the equations (12) which force all the $\Omega_{s}(\mathcal{C})$ to have the same exponential increase with $s$.

The $\mu_{n}$ can be easily calculated from the knowledge of $\lambda_{n}(x)$. The connective constant is given by

$$
\mu_{n}=\frac{1}{x_{n}}
$$

where $x_{n}$ is the smallest value of $x$ for which

$$
\lambda_{n}\left(x_{n}\right)=1 \text {. }
$$

The justification of (21) has been given by Klein [16] in the case of self avoiding walks. The generalization to lattice animals is straightforward. Let us give a simple proof of (21). For large $s$, the equations (12) become homogeneous

$$
\left(\mu_{n}\right)^{m(\mathcal{C})} a(\mathcal{C})=\sum_{\mathcal{C}^{\prime}} M\left(\mathcal{C}, \mathcal{C}^{\prime}\right) a\left(\mathcal{C}^{\prime}\right) .
$$

By looking at equations (8) and (9), one sees that (22) means exactly that the matrix $T$ has an eigenvalue $\lambda_{n}(x)=1$ for $x=1 / \mu_{n}$. The fact that all the coefficients $a(\mathcal{C})$ are positive implies that $\lambda_{n}(x)$ is the largest eigenvalue of the matrix $T$. Moreover, the $a(\mathcal{C})$ are the coefficients of the eigenvector of matrix $T$ corresponding to the eigenvalue $\lambda=1$ when $x=1 / \mu_{n}$.

In table I, we give the values of the $\mu_{n}$ that we found numerically by constructing the matrices $T$ for the four models A, B, C and D. The big surprise about these results is that for model $\mathrm{A}$, the $\mu_{n}$ have an exact

\begin{tabular}{|c|c|c|c|c|}
\hline $\mathrm{n}$ & mode $1 \mathrm{~A}$ & model $B$ & model C & mode1 D \\
\hline 2 & \multirow{11}{*}{$1+2 \cos \left(\frac{\pi}{2 n}\right)$} & 2. & 3.10068 & 1.83929 \\
\hline 3 & & 2.41421 & 3.30671 & 2.57499 \\
\hline 4 & & 2.54386 & 3.39005 & 2.98014 \\
\hline 5 & & 2.60450 & 3.43099 & 3.20168 \\
\hline 6 & & 2.63746 & 3.45390 & 3.33009 \\
\hline 7 & & 2.65736 & 3.46794 & 3.40943 \\
\hline 8 & & 2.67029 & 3.47715 & 3.46133 \\
\hline 9 & & 2.67916 & & 3.49694 \\
\hline 10 & & 2.68551 & & 3.52236 \\
\hline 11 & & 2.69021 & & \\
\hline 12 & & 2.69378 & & \\
\hline \multirow[t]{2}{*}{$n=\infty$} & 3 & 2.71261 & 3.50766 & 3.6280 \\
\hline & & \pm .00001 & \pm .00006 & \pm .0010 \\
\hline
\end{tabular}
expression for any value of $n$ :

$$
\mu_{n}=1+2 \cos \frac{\pi}{2 n} .
$$

We found this result by numerical studies. It can be checked by putting $\lambda=1$ in equations (16) to (18)

Table I. - The connective constants $\mu_{n}$ of strips of width $n$ with periodic boundary conditions for the four models $A, B, C$ and $D$. For model $A$, the $\mu_{n}$ have an exact expression. The extrapolated values for $n \rightarrow \infty$ were determined in section 4. 


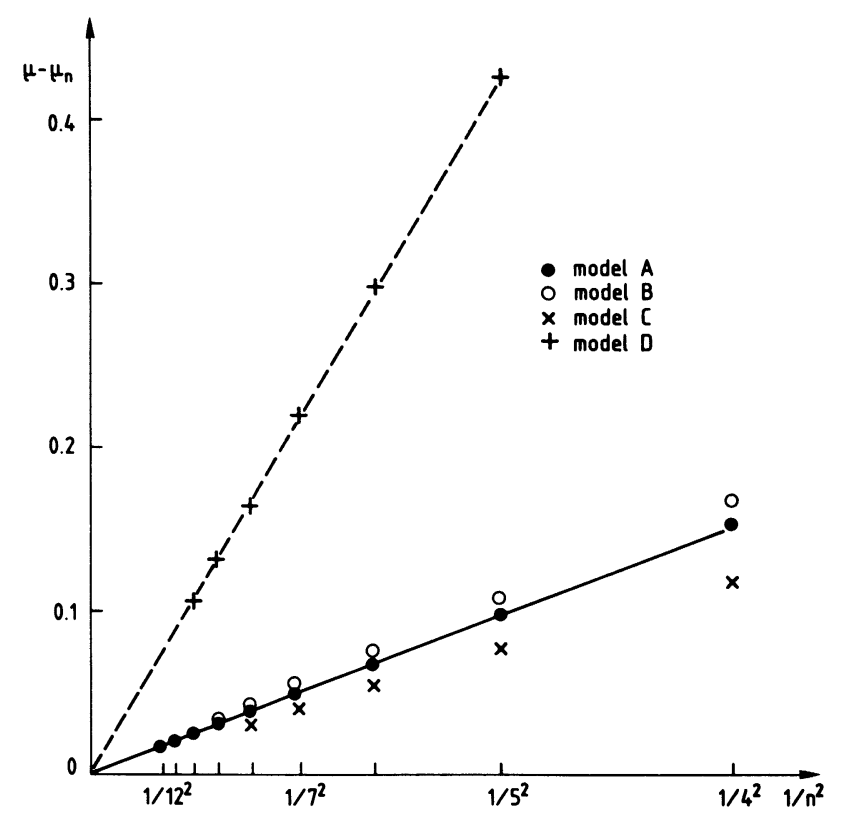

Fig. 2. - The differences $\mu-\mu_{n}$ versus $1 / n^{2}$ for the four models. The dashed line indicates the partially directed case.

for $n=2$ to 4 . For general $n$, it is possible to prove it and we shall give a proof in section 6 . For the three other models B, C, D, we did not find that the $\mu_{n}$ had a simple expression valid for all values of $n$.

In principle, one way to calculate the connective constant $\mu$ of the two dimensional system is to extrapolate the results of table I in the limit $n \rightarrow \infty$. However this extrapolation is less accurate than the extrapolation of the results of the phenomenological renormalization (section 4). It is why in table $I$, we compare the $\mu_{n}$ to the accurate estimations found in section 4.

In the four models, the $\mu_{n}$ seem to converge for large $n$ in the following way (see Fig. 2)

$$
\mu-\mu_{n} \sim \frac{1}{n^{2}} .
$$

In the problems of self-avoiding walks or of lattice animals, the connective constant plays the role of the critical temperature in usual statistical mechanics problems. The behaviour (24) can be compared with the shift of the critical temperature due to finite size effects $[17,18]$ : when the system is finite (of size $n$ ) on a few directions and infinite in the other directions (in order to have a critical temperature), the shift $(\Delta T)_{n}$ of the critical temperature is almost always [19] proportional to $n^{-1 / v}$. As we shall see in the next sections, in the problem of directed lattice animals, $v_{\perp}=\frac{1}{2}$ is presumably exact in two dimensions. Therefore (24) can be interpreted as $\mu-\mu_{n} \sim n^{1 / v_{\perp}}$. This is not surprising because $v_{\perp}$ governs the width of the animals which is the length to be compared to the finite width $n$ of the strip.

Another quantity which can be obtained from $\lambda_{n}(x)$ is the average length $L$ of the animals along the strip. For large $s$, this length behaves like

$$
L \sim b_{n} s
$$

because the strip is a one dimensional system.

Following the work of Klein [16] on self-avoiding walks, one can show that $b_{n}$ is given by :

$$
b_{n}=\left[x_{n} \frac{\mathrm{d} \lambda_{n}}{\mathrm{~d} x}\left(x_{n}\right)\right]^{-1}
$$

where $x_{n}$ is solution of equation (21).

We have calculated these $b_{n}$ for the four models $\mathrm{A}, \mathrm{B}$, C, D. Even for model A, we did not find a simple expression for these $b_{n}$. In principle, one expects that the $b_{n}$ increase for large $n$ like a power law with an exponent related to $v_{\|}$and $v_{\perp}$ via

$$
b_{n} \sim n^{\left(v_{\|}-1\right) / v_{\perp}}
$$

One can justify (27) by an argument similar to one used for polymers $[20,21]$. Consider a very long animal of $s$ sites on a strip of width $n$. One can divide the strip into adjacent rectangles of width $n$ and of length $n^{v_{\|} / v_{\perp}}$. At the scale of these rectangles, the animal is not affected by finite size effects. Therefore, the animal has $n^{1 / v_{\perp}}$ sites in each rectangle. It follows that the number of rectangles which are crossed by an animal of $s$ sites is proportional to $s / n^{1 / v_{\perp}}$ and the length of the animal is proportional to $\left(s / n^{1 / v_{\perp}}\right) n^{v_{\|} / v_{\perp}}$.

In figure 3 , we present a $\log -\log$ plot of $b_{n}$ versus $n$. This figure indicates that the three models $\mathrm{A}, \mathrm{B}, \mathrm{C}$ belong to the same universality class with $\left(1-v_{\|}\right) /$ $v_{\perp} \simeq 0.37$ whereas model $\mathrm{D}$ seems to have different exponents $\left(1-v_{\|}\right) / v_{\perp} \simeq 0.45$. This means that the partially directed animals may not be in the same universality class as the fully directed animals. This point will be discussed in the next section. One should notice that the power law behaviour (27) remains valid for very narrow strips and that we have in the $\log -\log$ plot a straight line from $n=3$ to $n=9$ or 13 depending on the model.

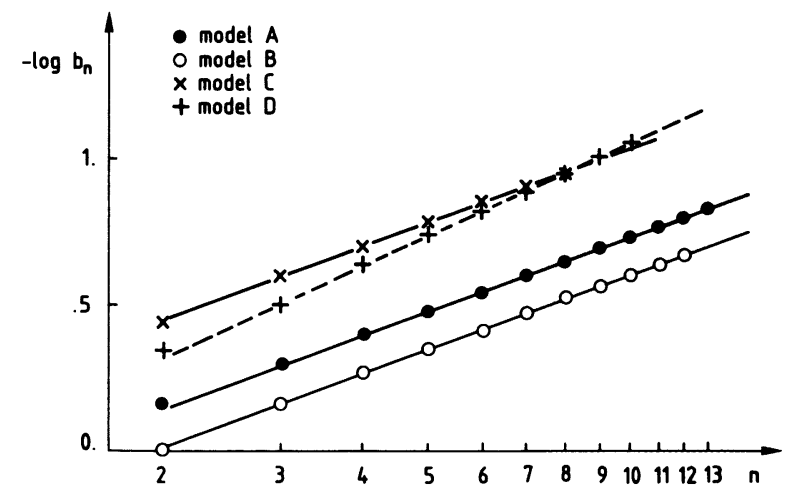

Fig. 3. - Plot of $\log \left(b_{n}\right)$ versus $\log n$ for the four models. The $b_{n}$ are defined by equation (25). The linear behaviours give the power laws (27). 
5. Phenomenological renormalization. - As it has been done in the case of directed percolation [5] and of non-directed animals [12], we have used the results obtained by transfer matrices to apply the phenomenological renormalization method [13]. The largest eigenvalue $\lambda_{n}(x)$ of the transfer matrix defines (as for non-directed animals [12]) a correlation length $\xi_{n}(x)$ :

$$
\exp \left(-\frac{1}{\xi_{n}(x)}\right)=\lambda_{n}(x)
$$

This length is the characteristic length along the strips and thus along the preferred direction. The phenomenological renormalization is based on a finite size scaling hypothesis : for large $n$ and when $x$ is in the neighbourhood of its critical value $x_{c}$ for the two-dimensional system, the $\xi_{n}(x)$ should satisfy :

$$
\xi_{n}(x) \sim n^{v_{\|} / v_{\perp}} F_{1}\left[n^{1 / \nu_{\perp}}\left(x-x_{c}\right)\right]
$$

where the function $F_{1}(z)$ is a regular function around $z=0$. This finite size scaling allows a sequence of estimations of the initial point $x_{\mathrm{c}}$, of the ratio $\varphi=v_{\|} / v_{\perp}$ and of the exponents $v_{\|}$and $v_{\perp}$ to be calculated. We determined the estimations $x_{\mathrm{c}}(n)$ of the critical point by choosing strips of three consecutive widths $n+1$, $n$ and $n-1$ and by defining $x_{\mathrm{c}}(n)$ as the solution of the following equation :

$$
\log \left[\frac{\xi_{n+1}(x)}{\xi_{n}(x)}\right]=\log \left[\frac{\xi_{n}(x)}{\xi_{n-1}(x)}\right] .
$$

The estimations of $\varphi(n), v_{\perp}(n)$ and $v_{\|}(n)$ were then found by

$$
\begin{aligned}
& \varphi(n)=\frac{\log \left[\xi_{n+1}\left(x_{\mathrm{c}}(n)\right) / \xi_{n}\left(x_{\mathrm{c}}(n)\right)\right]}{\log [(n+1) / n]} \\
& \frac{1}{v_{\perp}(n)}=-\varphi(n)+\frac{\log \left[\frac{\mathrm{d} \xi_{n+1}}{\mathrm{~d} x}\left(x_{\mathrm{c}}(n)\right) / \frac{\mathrm{d} \xi_{n}}{\mathrm{~d} x}\left(x_{\mathrm{c}}(n)\right)\right]}{\log [(n+1) / n]} \\
& v_{\|}(n)=\varphi(n) v_{\perp}(n)
\end{aligned}
$$

More details about this phenomenological renormalization method can be found in references $[5,12,13]$.

If the finite size scaling hypothesis were not only asymptotic in $n$ but also valid for narrow strips, the estimations $x_{\mathrm{c}}(n), v_{\perp}(n)$ and $v_{\|}(n)$ given by equations (30) to (33) would be equal to their exact values $x_{c}$, $v_{\perp}$ and $v_{\|}$. The simple idea followed here is that by increasing $n$, the estimations will be closer and closer to their exact values.

In figure 4, we have plotted the $x_{\mathrm{c}}(n)$ for the four models versus $n^{-4}$. The convergence is very regular and linear in $n^{-4}$ for the four models. It allows us to find accurate extrapolated values of $x_{\mathrm{c}}$ (see Table II).

For model A, our numerical results strongly support the conjecture $x_{\mathrm{c}}=\frac{1}{3}$ proposed in reference [11]

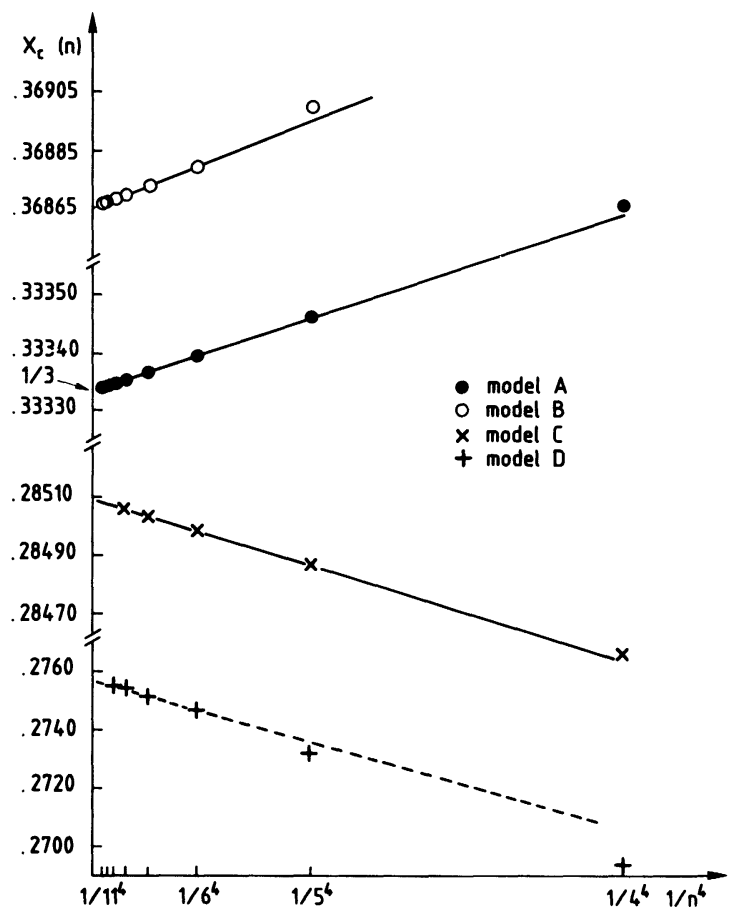

Fig. 4. - The values of $x_{\mathrm{c}}(n)$ solutions of (30) versus $n^{-4}$. The extrapolated values and the error bars are given in table II.

and which follows also from our observation of section 3 that $\mu_{n}=1+2 \cos \frac{\pi}{2 n}$. We shall establish this result in section 6 . For model $\mathrm{C}$, our extrapolated value $x_{\mathrm{c}}=0.28509 \pm 0.00001$ does not disagree too much with $x_{\mathrm{c}}=0.2849 \pm 0.0001$ found by Redner and Yang [10] although our estimation is more accurate.

In figures 5 and 6 , we have plotted the $v_{\|}(n)$ and $v_{\perp}(n)$. We see that for the models A, B and C, the

\begin{tabular}{|c|c|c|c|}
\hline model & $x_{c}$ & $v^{\prime} / I$ & $v_{\perp}$ \\
\hline A & $\begin{array}{r}.3333335 \\
\pm .0000010\end{array}$ & $\begin{array}{r}.8185 \\
\pm .0010\end{array}$ & $\begin{array}{r}.5000 \\
\pm .0005\end{array}$ \\
\hline B & $\begin{array}{r}.368649 \\
\pm .000002\end{array}$ & $\begin{array}{r}.8187 \\
\pm .0010\end{array}$ & $\begin{array}{r}.5005 \\
\pm .0010\end{array}$ \\
\hline C & $\begin{array}{r}.28509 \\
\pm .00001\end{array}$ & $\begin{array}{r}.8180 \\
\pm .0010\end{array}$ & $\begin{array}{r}.498 \\
\pm .001\end{array}$ \\
\hline D & $\begin{array}{r}.27563 \\
\pm .00010\end{array}$ & $\begin{array}{c}.815 \\
\pm .010 \\
\frac{9}{11} \approx .8182\end{array}$ & $\begin{array}{r}.483 \\
\pm .001\end{array}$ \\
\hline
\end{tabular}
convergence is linear in $n^{-2}$. Our extrapolated values (see Table II) agree again rather well with those found

Table II. - Estimations of the critical point $x_{\mathrm{c}}$ and of the exponents $v_{\|}$and $v_{\perp}$ found by extrapolating the results presented in figures 4,5 and 6. 


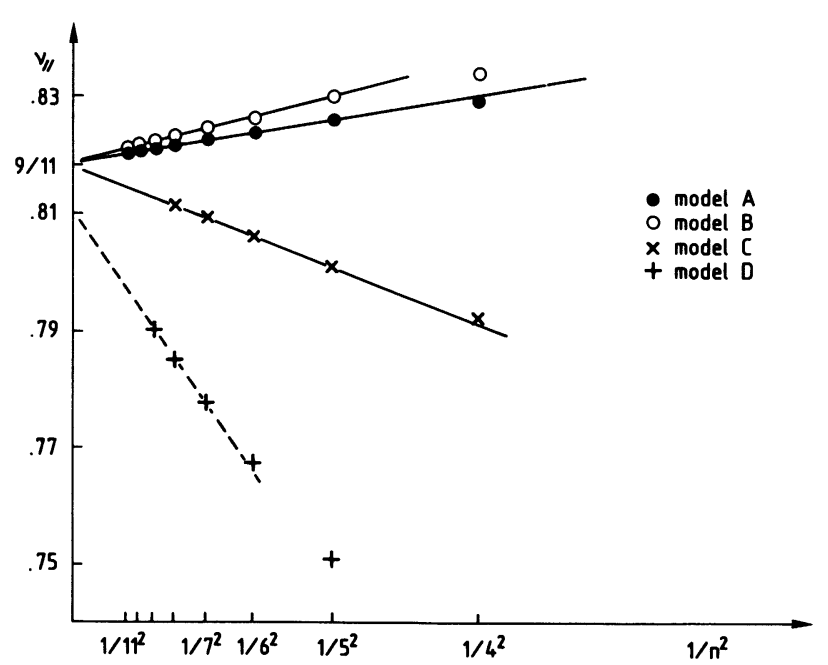

Fig. 5. - The estimations $v_{\|}(n)$ given by equation (33) versus $n^{-2}$. For models $\mathrm{A}, \mathrm{B}$ and $\mathrm{C}$, the results seem to converge very well to $9 / 11$. The extrapolated values are in table II.

by Redner and Yang [10]. For these three models, the values of $v_{\|}$and $v_{\perp}$ seem to be equal to

$$
\begin{aligned}
& v_{\perp}=\frac{1}{2} \\
& v_{\|}=\frac{9}{11} .
\end{aligned}
$$

For model $\mathrm{C}, v_{\perp}$ as found in table II is slightly different from $\frac{1}{2}$ but we do not believe that this difference is significant.

For model D, the convergence law is more complicated and our results are not very easy to extrapolate. However in figures 5 and 6 they do not seem to converge to values which agree with (34) and (35).

With the values of $v_{\|}$and $v_{\perp}$ given in table II, we

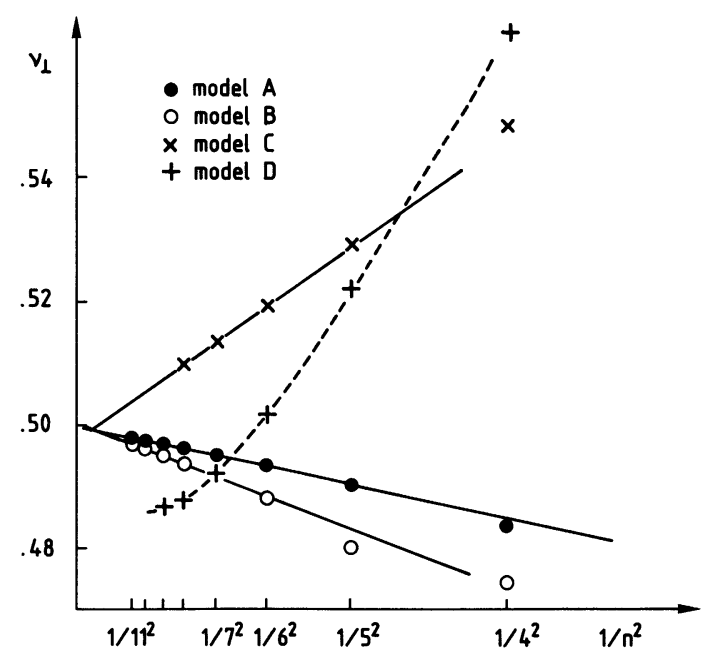

Fig. 6. - The estimations $v_{\perp}(n)$ given by equation (32) versus $n^{-2}$. Here the models A and B give $v_{\perp}=1 / 2$ and model $\mathrm{C}$ gives a slightly lower value. The extrapolated values are in table II. can come back to the slope $\left(1-v_{\|}\right) / v_{\perp}$ found in figure 3. For the first three models $\mathrm{A}, \mathrm{B}$ and $\mathrm{C}$, we find here $\left(1-v_{\|}\right) / v_{\perp}=0.363$ which agrees with 0.37 estimated in section 4 . On the contrary, for model $D$, table II gives $\left(1-v_{\|}\right) / v_{\perp}=0.38 \pm 0.02$ which is rather far from 0.45 found in the previous section. We think that in model $\mathrm{D}$, the small-size effects are more important than for the three other models. This would be responsible for the complicated convergence of $v_{\|}(n)$ and $v_{\perp}(n)$ and for the disagreement between the two estimations of $\left(1-v_{\|}\right) / v_{\perp}$. Therefore we think that our results of section 4 and of table II do not allow us to conclude that model $\mathrm{D}$ belongs to a different universality class.

The numbers given in table II can be compared with the predictions $[23,24]$ of the Flory theory in $d=2: v_{\|}=13 / 16=0.8125$ and $v_{\perp}=9 / 16=0.5625$. This shows that the Flory theory is a good approximation but is not exact in $d=2$.

Using the finite size scaling, we can also determine the exponent $\theta$ defined in equation (1). We have seen (eq. (15)) that one can calculate on a strip the generating functions $H(x, \mathrm{C})$ of the number of directed animals with a given root $\mathcal{C}$. To simplify the notation, let us define $h_{n}(x)$ by

$$
h_{n}(x)=H(x, \overline{\mathrm{C}})
$$

where the index $n$ is the width of the strip and $\overline{\mathrm{C}}$ is the root with only one site occupied.

For the two dimensional model, the generating function of directed animals with a one-site root is

$$
h_{\infty}(x)=\lim _{n \rightarrow \infty} h_{n}(x) \text {. }
$$

The exponent $\theta$ is related to the singularity of $h_{\infty}(x)$

$$
h_{\infty}(x) \sim\left|x-x_{\mathrm{c}}\right|^{1-\theta} .
$$

For the function $h_{n}(x)$, one can also write a finite size scaling hypothesis :

$$
h_{n}(x) \sim n^{(1-\theta) / v_{\perp}} F_{2}\left[n^{1 / v_{\perp}}\left(x-x_{\mathrm{c}}\right)\right]
$$

where $F_{2}(z)$ is again regular near $z=0$.

One can then find sequences of estimations $\chi(n)$ of the ratio $\chi=\frac{1-\theta}{v_{\perp}}$ by

$$
\chi(n)=\frac{\log \left[h_{n+1}\left(x_{\mathrm{c}}(n)\right) / h_{n}\left(x_{\mathrm{c}}(n)\right)\right]}{\log [(n+1) / n]} .
$$

Although there is in principle no difficulty in calculating the $h_{n}(x)$ by equations (15), it took us more computer time to calculate these $h_{n}(x)$ than the $\xi_{n}(x)$. This is why we have restricted our calculations of the $\chi(n)$ to the models $\mathrm{A}$ and $\mathrm{C}$. For model $\mathrm{A}$ we find for the extrapolated value of $\chi=0.985 \pm 0.005$ whereas for model $C$ we find $\chi=1 \pm 0.015$. Therefore we think that these results together with equation (34) agree rather well with $\theta=\frac{1}{2}$ conjectured in reference [11]. 
At the end of this section where we have presented our main numerical results, we have to say that there are a number of methods for finding estimations of the critical point and of the exponents by using finite size scaling. Each method can also be slightly modified in different ways. Here we have used the phenomenological renormalization for periodic boundary conditions which gives often very accurate results. All the slight modifications (changing $(n+1)$ into $(n-1)$ in equations (31) and (32); free boundary conditions) that we tried did not change our extrapolated results in a significant way.

6. Exact results and conjectures for model A. By studying numerically the model $A$, we noticed several simple features. First, as mentioned in section 4 , we found that $\mu_{n}=1+2 \cos (\pi / 2 n$ ) (see eq. (23)). Then we realized that the $a(\mathcal{C})$ which are solutions of the linear equations (22) have simple expressions when $\mu=\mu_{n}$ on a strip of width $n$. Lastly we noticed that a general formula seems to give the number $\Omega_{s}(\mathcal{C})$ of directed animals of $s$ sites for any root $\mathcal{C}$ and on a strip of any width $n$. This formula generalizes the conjecture of Dhar, Phani and Barma [11] to the case of strips of finite width and to any root $\mathrm{C}$.

We could only prove that the $\mu_{n}$ were given by (23) and that the expression of the $a(\mathcal{C})$ proposed below is correct. For the general formula of $\Omega_{s}(\mathcal{C})$, we did not succeed in finding a proof, even though all our numerical verifications indicate that our formula is correct.

Let us define a sequence $f_{n}$ of polynomials of the variable $\mu$ :

$$
\begin{aligned}
f_{0}(\mu) & =1 \\
f_{1}(\mu) & =\mu \\
f_{n+1}(\mu) & =(\mu-1) f_{n}(\mu)-f_{n-1}(\mu) .
\end{aligned}
$$

We found numerically that the $a(\mathcal{C})$ which are solutions of (22) :

$$
(\mu)^{m(\mathcal{C})} a(\mathcal{C})=\sum_{\mathcal{C}^{\prime}} M\left(\mathcal{C}, \mathfrak{C}^{\prime}\right) a\left(\mathfrak{C}^{\prime}\right)
$$

have the following expression when $\mu=\mu_{n}$

$$
a(\mathcal{C})=c\left(f_{1}\right)^{N_{1}}\left(f_{2}\right)^{N_{2}} \ldots\left(f_{n-1}\right)^{N_{n-1}}
$$

where $c$ is a constant (the same for all the $a(\mathcal{C})$ ), the $f_{i}$ are defined by (41) and the $N_{i}$ are the number of holes of $i$ sites in the root $\mathcal{C}$. (A hole of $i$ sites in $\mathcal{C}$ means that there are $i$ consecutive empty sites in the root (see Fig. 7).) A remarkable feature of the expression (42) is that it does not depend on the relative positions of the different holes in the root.

We are now going to prove that (42) gives a solution of (22) when $\mu=\mu_{n}$. Let us choose for the $a(\mathcal{C})$ the expression (42) for any value of $\mu$. Then define $b(\mathcal{C})$ by :

$$
b(\mathcal{C})=\sum_{\mathcal{C}^{\prime}} M\left(\mathcal{C}, \mathcal{C}^{\prime}\right) a\left(\mathcal{C}^{\prime}\right)
$$

The following proposition is true.

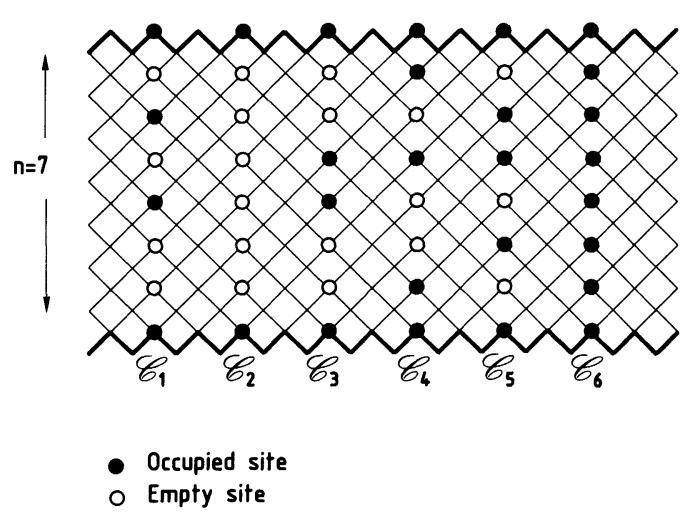

Fig. 7. - Example of configurations on a strip of width 7. The two heavy lines must be identified because of the periodic boundary conditions. Formula (42) gives here $a\left(\mathcal{C}_{1}\right)=c f_{1}^{2} f_{2}$, $a\left(\mathcal{C}_{2}\right)=c f_{6}, a\left(\mathcal{C}_{3}\right)=c f_{2}^{2}, a\left(\mathcal{C}_{4}\right)=c f_{1} f_{2}, a\left(\mathcal{C}_{5}\right)=c f_{1}^{3}, a\left(\mathfrak{C}_{6}\right)=c$. The horizontal arrow indicates the preferred direction.

Proposition. - For any value of $\mu$, and for any configuration $\mathrm{C}$, if the $a(\mathrm{C})$ are given by (42), then one has :

$$
b(\mathcal{C})=\mu^{m(\mathcal{C})} a(\mathcal{C})+c\left[f_{n-1}(\mu)-f_{n}(\mu)\right] .
$$

The proof of this proposition is rather long and we prefer to present it in appendix B. It follows from (44) that for all values of $\mu$ which satisfy

$$
f_{n-1}(\mu)-f_{n}(\mu)=0
$$

the $a(\mathcal{C})$ given by (42) are solutions of (22).

The $f_{p}$ can be written in another way. If we define $\alpha$ by

$$
\mu=1+2 \cos \alpha
$$

then, the $f_{p}$ become

$$
f_{p}(\mu)=\frac{\sin \left(p+\frac{1}{2}\right) \alpha}{\sin \frac{\alpha}{2}} .
$$

It is then clear from (45) that for all the values of $\mu$ of the form

$\mu=1+2 \cos \left[\frac{2 p+1}{2 n} \pi\right]$ with $0 \leqslant p \leqslant n-1$

the $a(\mathcal{C})$ defined by (42) are solutions of (22). However it is only if

$$
\mu=\mu_{n}=1+2 \cos \left(\frac{\pi}{2 n}\right)
$$

that all the $a(\mathcal{C})$ are positive (see eqs. (42) and (48)), i.e. the largest eigenvalue $\lambda_{n}(x)$ of the transfer matrix is equal to 1 . 
We have proved that $\mu_{n}=1+2 \cos (\pi / 2 n)$ for a strip of width $n$ and that the $a(\mathcal{C})$ solutions of (22) are given by the expression (42).

Let us now come to the conjecture for the number of animals on a strip of width $\boldsymbol{n}$. First, for the number $\Omega_{s}(\overline{\mathcal{C}})$ of animals with a root composed of a single occupied site, we propose

$$
\Omega_{s}(\overline{\mathcal{C}})=\frac{1}{n} \sum_{p=0}^{n-1}\left(1+\cos \alpha_{p}\right)\left(1+2 \cos \alpha_{p}\right)^{s-1}
$$

where

$$
\alpha_{p}=\frac{2 p+1}{2 n} \pi .
$$

For a more general root $\mathcal{C}$ we propose

$$
\begin{aligned}
\Omega_{s}(\mathcal{C}) & =\frac{1}{n} \sum_{p=0}^{n-1}(-)^{p} \sin \alpha_{p} \times \\
& \times \prod_{i=1}^{n-1}\left(\frac{\sin \left(i+\frac{1}{2}\right) \alpha_{p}}{\sin \frac{\alpha_{p}}{2}}\right)^{N_{i}}\left(1+2 \cos \alpha_{p}\right)^{s-1}
\end{aligned}
$$

where $\alpha_{p}$ are given by (50) and the $N_{i}$ were defined in formula (42).

In the limit $n \rightarrow \infty$, the sums in (49) and (51) become integrals and we recover the conjecture of Dhar, Phani and Barma [11].

If for a root $\mathcal{C}$ which has $N_{p}$ holes of size $p$ (eq. 42) we denote $\Omega_{s}(\mathrm{C})$ by $\Omega_{s}\left(N_{1}, N_{2}, \ldots, N_{p}, \ldots\right)$, then expression (51) leads to a simple recurrence relation between animals of different sizes :

$$
\begin{aligned}
\Omega_{s+1}\left(\ldots, N_{k-1}, N_{k},\right. & \left.N_{k+1}, \ldots\right)= \\
= & \Omega_{s}\left(\ldots, N_{k-1}, N_{k}, N_{k+1}+1, \ldots\right)+ \\
& +\Omega_{s}\left(\ldots, N_{k-1}, N_{k}+1, N_{k+1}, \ldots\right) \\
& +\Omega_{s}\left(\ldots, N_{k-1}+1, N_{k}, N_{k+1}, \ldots\right) .
\end{aligned}
$$

In all the numerical calculations we did, the expressions (49) and (51) were correct. Nevertheless, we did not succeed in proving them. If we assume that (49) is correct, we find for $h_{n}(x)$ defined in equation (36)

$$
h_{n}(x)=\frac{x}{n} \sum_{p=0}^{n-1} \frac{1+\cos \alpha_{p}}{1-x\left(1+2 \cos \alpha_{p}\right)} .
$$

For $n$ large and $x$ close to $1 / 3$, one finds that

$$
h_{n}(x) \simeq n F_{2}\left[n^{2}\left(\frac{1}{3}-x\right)\right]
$$

with

$$
F_{2}(z)=\frac{8}{\pi^{2}} \sum_{p=0}^{\infty} \frac{1}{\frac{36}{\pi^{2}} z+(2 p+1)^{2}} .
$$

This is exactly the finite size scaling expression (39) with $v_{\perp}=\frac{1}{2}$ and $\theta=\frac{1}{2}$.

At the end of this section, we have to underline that formula (42) gives the eigenvector of the transfer matrix for $\mu=\mu_{n}$, i.e. $x=[1+2 \cos (\pi / 2 n)]^{-1}$.
For other values of $x$, the eigenvector is much more complicated and we did not find any way to generalize (42). It would be interesting to know more on these eigenvectors of the transfer matrix if one wants to find the exact value of $v_{\|}$.

Acknowledgments. - We are grateful to D. Bessis, E. Brézin, W. Kinzel, A. Pandey and Y. Pomeau for constructive suggestions. We would also like to thank A. R. Day and T. C. Lubensky for stimulating discussions.

\section{APPENDIX A}

In this appendix, we give an example to illustrate the general method described in section 3 . We write the transfer matrix for a strip of width $n=4$ in the case of model A. In figure 8, we have represented a strip of width 4 with periodic boundary conditions and the 5 different configurations $\mathcal{C}$ which remain when the translational symmetry has been used.

If we simplify the notation of section 3 a little by writing

$$
\psi_{R}(i)=\psi_{R}\left(C_{i}, x\right)
$$

the matrix $M$ can be written

$$
\begin{aligned}
\psi_{R+1}(1)=x\left[2 \psi_{R}(1)+\right. & \left.\psi_{R}(2)\right] \\
\psi_{R+1}(2)=x^{2}\left[3 \psi_{R}(1)+\right. & \left.2 \psi_{R}(2)+\psi_{R}(3)+\psi_{R}(4)\right] \\
\psi_{R+1}(3)=x^{2}\left[4 \psi_{R}(1)+\right. & 4 \psi_{R}(2)+2 \psi_{R}(3)+ \\
& \left.+4 \psi_{R}(4)+\psi_{R}(5)\right] \\
\psi_{R+1}(4)=x^{3}\left[4 \psi_{R}(1)+\right. & 4 \psi_{R}(2)+2 \psi_{R}(3)+ \\
& \left.+4 \psi_{R}(4)+\psi_{R}(5)\right] \\
\psi_{R+1}(5)=x^{4}\left[4 \psi_{R}(1)+\right. & 4 \psi_{R}(2)+2 \psi_{R}(3)+ \\
& \left.+4 \psi_{R}(4)+\psi_{R}(5)\right] .
\end{aligned}
$$

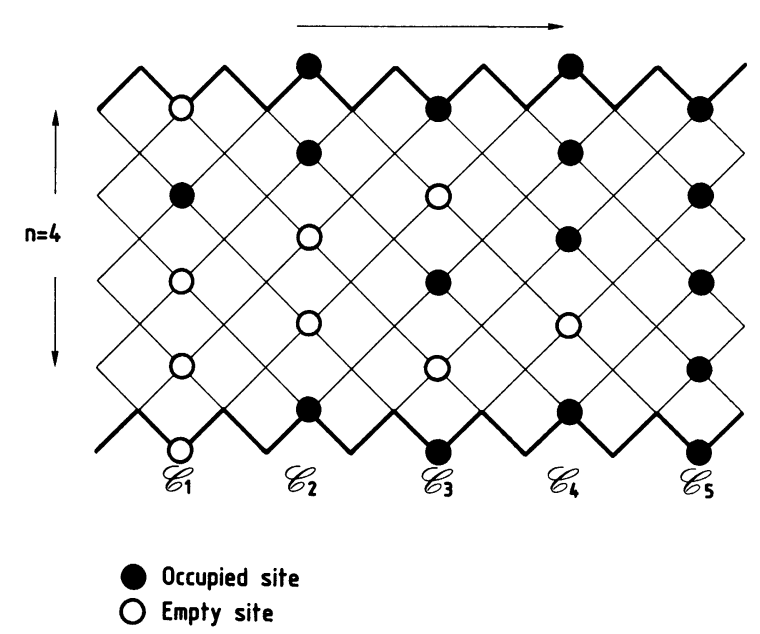

Fig. 8. - To realize a strip of width 4 with periodic boundary conditions the two heavy lines must be identified. We have drawn the five different configurations $\mathcal{C}_{i}$ which are involved in writing the transfer matrix. The horizontal arrow indicates the preferred direction. 
Taking advantage of the fact that

$$
\psi_{R}(5)=x \psi_{R}(4)=x^{2} \psi_{R}(3) .
$$

One finds that the largest eigenvalue $\psi_{4}(x)$ of the transfer matrix for a strip of width 4 is the largest root of :

$$
\begin{aligned}
& \lambda^{3}-\lambda^{2}\left(x^{4}+4 x^{3}+4 x^{2}+2 x\right)+ \\
& +\lambda\left(2 x^{6}+6 x^{5}+8 x^{4}+5 x^{3}\right)-x^{7}+2 x^{5}=0 .
\end{aligned}
$$

\section{APPENDIX B}

In this appendix we prove some exact relations for model A (site animals on a square lattice with both axes oriented, see figure $1 a$ ), when the animals are drawn on strips of width $n$, with periodic boundary conditions. These relations are used in the article to show that the growth parameter $\mu_{n}$ on a strip has the simple form $\mu_{n}=1+2 \cos (\pi / 2 n)$.

For a large number of sites $s$, the number $\Omega_{s}(\mathrm{C})$ of animals with root $\mathcal{C}$ behaves like

$$
\Omega_{s}(\mathbb{C}) \sim \mu_{n}^{s} a(\mathbb{C})
$$

and it was shown in section 6 that the $2^{n}-1$ quantities $a(\mathcal{C})$ are solutions of the system of linear equations

$$
\mu_{n}^{m(\mathcal{C})} a(\mathrm{C})=\sum_{\mathrm{C}^{\prime}}^{\prime} a\left(\mathrm{C}^{\prime}\right)
$$

where $m(\mathcal{C})$ is the number of occupied sites in the root $\mathcal{C}$ and the prime on $\Sigma^{\prime}$ denotes the sum over all $\mathrm{C}^{\prime}$ which can follow $\mathcal{C}$ (called its successors).

The second member of equation (22) was denoted by $b(\mathcal{C})$, that is :

$$
b(\mathcal{C})=\sum_{\mathbb{C}^{\prime}}^{\prime} a\left(\mathcal{C}^{\prime}\right)
$$

for arbitrary values of the $a(\mathcal{C})$. Here we shall prove that for any number $\mu:$ if

$$
a(\mathcal{C})=c \prod_{j=1}^{n-1} f_{j}^{N_{j}}
$$

where $c$ is a constant and $f_{j}(\mu)$ is the $j$ th polynomial of the sequence defined by :

$$
f_{0}(\mu)=1, \quad f_{1}=\mu, \quad f_{j+1}=(\mu-1) f_{j}-f_{j-1}
$$

then

$$
b(\mathcal{C})=\mu^{m(\mathcal{C})} a(\mathcal{C})+c\left(f_{n-1}(\mu)-f_{n}(\mu)\right) .
$$

An essential step of the proof is the remark that, within hypothesis (42), the quantities which are very simple are sums of $a(\mathrm{C})$ over all $\mathrm{C}$ belonging to some set of roots. This set may for example contain all the $2^{n-2}$ roots with two given sites occupied, any other site being either occupied or not.
In part 1 we introduce some definitions and notations and derive a relation for the number of successors of a given configuration, in terms of sums over particular sets of roots. In part 2 , we draw some consequences of the factorization assumption (42) and find a simple expression for the sums appearing in part 1. Finally in part 3, we show that these sums may be rearranged to yield equation (44).

1. A relation for the sum on the successors. $-\mathrm{We}$ first show a useful relation for $b(\mathcal{C})$, which is a consequence of the so-called method of inclusion and exclusion, and is related to the usual theorem in probability theory for the probability of the union of $M$ events. This theorem [22] says that the probability of the realization of at least one among the $M$ events $A_{1}, A_{2}, \ldots, A_{M}$, that is of $A=A_{1} \cup A_{2} \cup \ldots \cup$ $A_{M}$, is given by

$$
P(A)=S_{1}-S_{2}+\cdots+(-1)^{M-1} S_{M}
$$

where $S_{p}$ is the sum of the probabilities of $A_{i_{1}} \cap$ $A_{i_{2}} \cap \ldots \cap A_{i_{p}}$, over all possible choices of $p$ events $\left\{A_{i_{1}}, \ldots, A_{i_{p}}\right\}$ with $1 \leqslant i_{1} \leqslant i_{2}<\cdots<i_{p} \leqslant M$.

We choose to number the sites of a column on a strip of width $n$ from 1 to $n$. Due to the periodic boundary conditions, one has to remember that sites 1 and $n$ are adjacent.

We are interested in the quantity

$$
b(\mathcal{C})=\sum_{\mathcal{C}^{\prime}}^{\prime} a\left(\mathcal{C}^{\prime}\right)
$$

where the summation is over the successors of $\mathcal{C}$. The only constraint for $\mathcal{C}^{\prime}$ to be a successor of $\mathcal{C}$ is that any site of $\mathfrak{C}^{\prime}$ linked to two empty sites of $\mathcal{C}$ has to be empty (see Fig. 9).
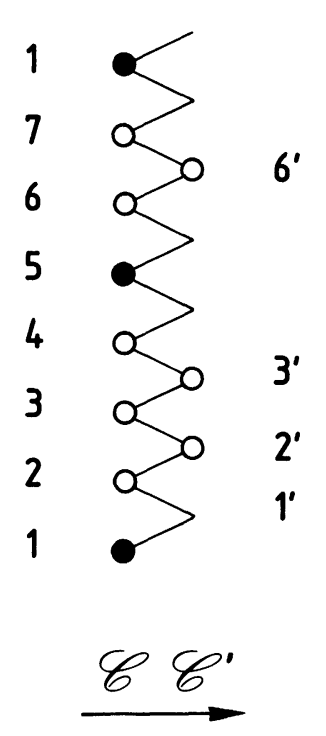

Fig. 9. - Typical configuration $\mathbb{C}$ with the set $\mathcal{g}(\mathcal{C})$ of sites which are forbidden in any $\mathcal{C}^{\prime}$ successor of $\mathcal{C}$. Here $\mathcal{H}(\mathcal{C})=$ $\left\{2^{\prime}, 3^{\prime}, 6^{\prime}\right\} . \mathcal{F}(\mathcal{C})$ is the union of two non adjacent subsets : $\mathcal{f}(\mathcal{C})=\left\{2^{\prime} 3^{\prime}\right\} \cup\left\{6^{\prime}\right\}$. 
The other sites of $\mathrm{C}^{\prime}$ may be either empty or occupied. Then $b(\mathcal{C})$ is the sum over all the $2^{n}-1$ roots, minus the sum of $a\left(\mathrm{C}^{\prime}\right)$ over the set $A$ of all $\mathrm{C}^{\prime}$ in which at least one among the forbidden sites is occupied. To obtain this last sum, we can follow the same reasoning that leads to $P(A)$ on (B.1) where " the sum of $a(\mathcal{C})$ over $\mathcal{C}$ belonging to a given subset" replaces " the probability of a given event ".

We denote by $I(\mathcal{C})$ the set of these forbidden sites in any successor of $\mathcal{C}$ and $M \equiv \operatorname{Card} I(\mathcal{C})$. If $I(\mathcal{C})=$ $\left\{l_{1}, l_{2}, \ldots, l_{M}\right\}$ with $1 \leqslant l_{1}<l_{2}<\cdots<l_{M} \leqslant n$ and if $A_{i}$ is the set of roots in which the site $i$ is occupied (any other site being either occupied or not) then the set $A$ of roots which are not successors of $\mathcal{C}$ is

$$
A=\bigcup_{k=1}^{M} A_{l_{k}} .
$$

If we call $A_{i, j, k, \ldots}$ the set of roots for which sites $i, j, k, \ldots$ are occupied and $\alpha(\{i, j, k, \ldots\})$ the sum of $a(\mathcal{C})$ over the roots of $A_{i, j, k, \ldots}$, one has :

$$
\begin{gathered}
A_{i, j, k, \ldots}=A_{i} \cap A_{j} \cap A_{k} \cdots \\
\alpha(\{i, j, k \ldots\})=\sum_{\mathcal{C} \in A_{i, j, j} \ldots} a(\mathcal{C})
\end{gathered}
$$

and

$$
\alpha(\phi)=\sum_{\text {alle }} a(\mathcal{C})
$$

$\phi$ denotes the empty set.

Then $b(\mathcal{C})$ can be obtained by

$$
b(\mathcal{C})=\sum_{p=0}^{M}(-)^{p}\left[\sum_{\left\{\mathscr{\gamma}_{p}\right\}} \alpha\left(\mathfrak{f}_{p}\right)\right]
$$

where $g_{p}=\left\{i_{1}, i_{2}, \ldots, i_{p}\right\}$ denotes here and in the following any subset $\left(1 \leqslant i_{1}<i_{2}<\cdots i_{p} \leqslant n\right)$ included in $I(\mathcal{C})$.

Note that (B.7) is a direct consequence of theorem (B.1) by defining a function $P$ on the set whose elements are the subsets of roots by

$$
P(\mathcal{E})=\left[\sum_{\mathcal{C} \in \mathcal{E}} a(\mathcal{C})\right] / \sum_{\text {all } \mathcal{E}} a(\mathcal{C})
$$

which plays the role of a measure.

2. Factorization hypothesis and consequences Let $\mu$ be a real number and $\left\{f_{j}(\mu)\right\}$ be the sequence of polynomials defined by (41). Inspired by our numerical findings, we now make the factorization hypothesis that for every root $\mathcal{C}$

$$
a(\mathcal{C})=c \prod_{j=1}^{n-1}\left(f_{j}\right)^{N_{j}} .
$$

Note that (42) defines $a(\mathcal{C})$ as a function of $\mathcal{C}$ and of the variable $\mu$. One should notice that (42) implies that $a(\mathcal{C})$ does not depend on the relative positions of the holes in the root $\mathrm{C}$. For simplicity, in the following, we take $c=1$.

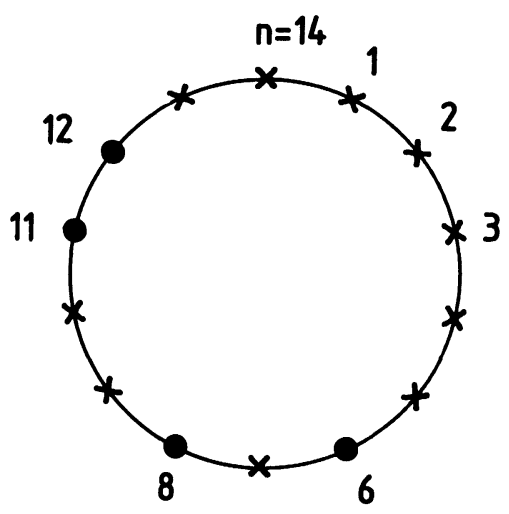

Fig. 10. - Typical set $\mathcal{C}$ placed on a column with periodic boundary conditions : $\mathfrak{F}=\{6,8,11,12\}, N(\mathcal{F})=3$.

The result we want to prove in this part is that with this particular choice for $a(\mathcal{C})$, the quantities $\alpha(\{i, j, \ldots\})$ defined in (B.5) are very simple. We are going to show that for any set $y_{p}$ of $p$ sites, one has : if $1 \leqslant p \leqslant-1$ :

$$
\alpha\left(\mathfrak{g}_{p}\right)=\mu^{n-p-N}(1+\mu)^{N}
$$

where $N=N\left(\mathfrak{g}_{p}\right)$ is the number of holes (of size $\geqslant 1$ ) between the sites belonging to $g_{p}$ (see Fig. 10) and if $p=0$

$$
\begin{aligned}
\alpha(\phi) & =\mu^{n}+f_{n-1}(\mu)-f_{n}(\mu) \\
& =(1+\mu)^{N(\phi)} \mu^{n-N(\phi)}+f_{n-1}(\mu)-f_{n}(\mu)
\end{aligned}
$$

with $N(\phi)=0$.

To prove (B. 8a) and (B.8b), we need to establish some relations about the consequences of (42).

Relation 1 . - If we define $\mathrm{C}_{j}$ as the root with only one hole of size $j$, for $0 \leqslant j \leqslant n-1$, then

$$
a\left(\mathfrak{C}_{j}\right)=f_{j} .
$$

This is an obvious consequence of (42).

Relation 2. - If $\mathcal{C}$ is a root with a hole (and possibly others) of size $p$, from (42) $a(\mathcal{C})$ contains a factor $f_{p}$. Then one can write

$$
a(\mathrm{C})=f_{p} a\left(\mathrm{C}^{*}\right)
$$

where $\mathcal{C}^{*}$ is the root obtained from $\mathcal{C}$ by filling the hole. More generally, if $\mathcal{C}$ contains $k$ holes of sizes $p_{1}, p_{2}, \ldots, p_{k}$ (and possibly others), one has

$$
a(\mathrm{C})=\left(f_{p_{1}} f_{p_{2}} \ldots f_{p_{k}}\right) a\left(\mathrm{C}^{*}\right)
$$

where $\mathrm{C}^{*}$ is the root obtained from $\mathrm{C}$ by filling the $k$ holes (see Fig. 11).

Relation 3. - Let $\Lambda$ be a set of roots having in common the sites $l$ for $l \leqslant i$ and $l \geqslant i^{\prime}\left(i<i^{\prime}\right)$ with $i$ and $i^{\prime}$ occupied. Suppose that these common sites contain (exactly) $k$ holes $(k \geqslant 0)$, say of sizes $p_{1}, \ldots, p_{k}$. 


$\begin{array}{rrrrl}n \bullet & \bullet & \bullet & \bullet & \bullet \\ 0 & \bullet & 0 & \bullet & 0 \\ \mathrm{i}^{\bullet} & \bullet & \mathrm{i} \bullet & \bullet & \bullet \mathrm{i}^{\prime} \\ \bullet & \bullet & 0 & 0 & \bullet \\ 0 & 0 & 0 & 0 & \bullet \\ \mathrm{i} \bullet & \bullet & \mathrm{i} \bullet & \bullet & \bullet \mathrm{i} \\ 0 & \bullet & 0 & \bullet & 0 \\ 10 & \bullet & 0 & \bullet & 0 \\ \mathscr{C}_{\mathrm{a}} & \mathscr{C}_{\mathrm{a}} & \mathscr{C}_{\mathrm{b}} & \mathscr{C}_{\mathrm{b}}^{*} & \mathscr{C}_{\mathrm{A}}\end{array}$

Fig. 11. $-\mathrm{C}_{\mathrm{a}}$ and $\mathrm{C}_{\mathrm{b}}$ have in common the sites $l$ for $l \leqslant i$ and $l \geqslant i^{\prime} . \mathrm{C}_{\mathrm{a}}^{*}$ and $\mathrm{C}_{\mathrm{b}}^{*}$ are the roots obtained from $\mathrm{C}_{\mathrm{a}}$ and $\mathrm{C}_{\mathrm{b}}$ by filling these sites. $\mathcal{C}_{A}$ is the root obtained (from $\mathrm{C}_{a}$ or $\mathcal{C}_{b}$ ) by filling the sites $l, i \leqslant l \leqslant i^{\prime}$.

Hence they contribute in $a(\mathcal{C})$ for any $\mathcal{C}$ of $\Lambda$ for the same factor $E_{A}$ :

$$
E_{\Lambda}=f_{p_{1}} f_{p_{2}} \ldots f_{p_{k}} .
$$

If $\mathfrak{C}_{A}$ is the configuration obtained (from any $\mathcal{C}$ of $\Lambda$ ) by filling all sites $l$ for $i \leqslant l \leqslant i^{\prime}$, one can see from (42) that (Fig. 11) :

$$
E_{A}=a\left(\mathcal{C}_{A}\right) .
$$

Then, if $\Lambda^{*}$ is the set of roots obtained from $\Lambda$ by filling all sites for $l \leqslant i$ and $l \geqslant i^{\prime}$, one has the relation

$$
\sum_{\mathcal{C} \in \boldsymbol{A}} a(\mathrm{C})=a\left(\mathrm{C}_{\boldsymbol{A}}\right) \sum_{\mathrm{C}^{*} \in \Lambda^{*}} a\left(\mathrm{C}^{*}\right) \text {. }
$$

Relation 4. - We have yet defined $A_{i j}$ as the set of all the roots having the sites $i$ and $j(i<j)$ occupied, every other site being either occupied or empty. The sum of the $a(\mathrm{C})$ over all the roots $\mathrm{C} \in A_{i j}$ was called $\alpha(\{i, j\})$.

From relation 3 , it follows that $\alpha(\{i, j\})$ can be written as

with

$$
\alpha(\{i, j\})=d_{q_{1}} d_{q_{2}}
$$

$$
d_{q_{1}}=\sum_{\mathcal{e} \in \Lambda_{1}} a(\mathrm{C})
$$

and

$$
d_{q_{2}}=\sum_{\mathrm{e} \in \Lambda_{2}} a(\mathcal{C})
$$

where $\Lambda_{1}$ is the set of roots with all the sites $l$ occupied for $l \leqslant i$ and $l \geqslant j$, the other sites $l(i+1 \leqslant l \leqslant j-1)$ being either occupied or empty. Similarly $\Lambda_{2}$ is the set of roots with all the sites $l$ occupied for $i \leqslant l \leqslant j$, the other sites being either occupied or empty (see Fig. 12). Due to the periodic boundary conditions $d_{q_{1}}$ and $d_{q_{2}}$ depend only on the two distances $q_{1}$ and $q_{2}$ between the two sites $i$ and $j$

$$
\begin{aligned}
& q_{1}=j-i-1 \\
& q_{2}=n-j+i-1 .
\end{aligned}
$$

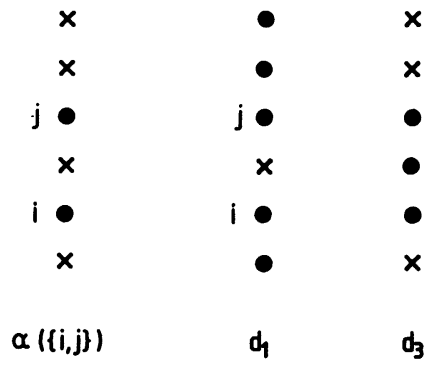

Fig. 12. - Sum of $a(\mathcal{C})$ for all $\mathcal{C}$ having the sites $i$ and $j$ occupied. A cross $(x)$ means the sum is on the two cases, site empty and occupied. One gets $\alpha(\{i, j\})=d_{1} d_{3}$.

We shall obtain the expression of the $d_{q}$ in the relations 6 and 7. One may note that if $i$ and $j$ are neighbours, that is $j=i+1, q_{1}$ is zero, and $\Lambda_{1}$ reduces to the set of the root $\mathrm{C}_{0}$. Then

$$
d_{0}=a\left(\mathfrak{C}_{0}\right)
$$

thus, by (B.9) : $d_{0}=1$.

Relation 5. - It is easy to see that (B.13) can be generalized to any set of sites $\gamma_{p}=\left\{i_{1}, i_{2}, \ldots, i_{p}\right\}$ with $1 \leqslant i_{1}<i_{2}<\cdots<i_{p} \leqslant n$

$$
\alpha\left(\mathfrak{g}_{p}\right)=d_{q_{1}} d_{q_{2}} \ldots d_{q_{p}}
$$

with

$$
\left.\begin{array}{l}
q_{j}=i_{j+1}-i_{j}-1 \text { if } 1 \leqslant j \leqslant p-1 \\
q_{p}=n-i_{p}+i_{1}-1
\end{array}\right\}
$$

Relation 6. - We are now going to calculate the $d_{q}$. We have yet defined $d_{q}$ as the sum of the $a(\mathcal{C})$ over every $\mathcal{C}$ having all its sites occupied except the sites 1 to $q$ which may be either empty or occupied. One has

$$
d_{0}=a\left(\mathcal{C}_{0}\right)=f_{0}=1
$$

which was shown in (B.18) and for $1 \leqslant q \leqslant n-1$ one can write

$$
\begin{aligned}
d_{q}=f_{q}+f_{q-1} & d_{0}+f_{q-2} d_{1}+\cdots+ \\
& +f_{i-1} d_{q-i}+\cdots+f_{0} d_{q-1} .
\end{aligned}
$$

In the right hand side of (B.22), the first term represents the root with all the $q$ sites empty. The second term represents the root with the first $(q-1)$ sites empty and the last site occupied. The term $f_{i-1} d_{q-i}$ represents more generally the sum over all the roots with the first $(i-1)$ sites empty, the $i$ th site occupied, the last $(q-i)$ sites being either empty or occupied. The recursion relation (B.22) allows to calculate $d_{q}$ knowing $d_{q-1}, d_{q-2}, \ldots, d_{0}$.

For $q=1$ (B.22) gives

$$
d_{1}=f_{1}+f_{0}=\mu+1 .
$$


Assuming that $d_{p}=\mu^{p-1}(1+\mu)$ for $1 \leqslant p \leqslant q-1$, and using the fact that $d_{0}=1$, one can write (B.22) as

$$
\begin{array}{r}
d_{q}-\mu d_{q-1}=f_{q}+f_{q-1} d_{0}+f_{q-2} d_{1}-\mu f_{q-2}- \\
-\mu f_{q-1} .
\end{array}
$$

Using the recursion relation (41), one gets

$$
d_{q}=\mu d_{q-1}
$$

and this proves that

$\left.\begin{array}{l}d_{0}=1 \\ d_{q}=\mu^{q-1}(1+\mu) \text { for } 1 \leqslant q \leqslant n-1\end{array}\right\}$

The recursion relation (B.24) is modified for $d_{n}$. Due to the periodic boundary conditions it becomes $d_{n}=d_{n-1}+\sum_{q=0}^{n-2} q f_{q} d_{n-q-2}+(n-1) f_{n-1}$.

This relation can be understood by choosing a particular site. The sum over all the roots for which this site is occupied gives $d_{n-1}$. More generally, the sum over all the roots for which this particular site is empty and belongs to a hole of size exactly $q$ is $q f_{q} d_{n-q-2}$. The factor $q$ comes from the $q$ possible positions of the particular site in the hole. The factor $f_{q}$ is the contribution of the hole and the factor $d_{n-q-2}$ comes from the sum over the $n-q-2$ sites which can be occupied or empty.

It is not too hard to show, using the relation (B.26) and the expression of the $d_{n}$ and of the $f_{n}$, that :

$$
d_{n}=\mu^{n}+f_{n-1}(\mu)-f_{n}(\mu) .
$$

One way to do that is to use the change of variable (46) and to write the $f_{p}$ as in (47). Then the sum can be done as the sum of a geometric series.

Proof of (B.8a) and (B.8b). - To prove (B.8a) we have just to combine relation 5 and (B.25):

$$
\alpha\left(\mathfrak{f}_{p}\right)=d_{p_{1}} d_{p_{2}} \ldots d_{p_{q}}
$$

then

$$
\alpha\left(\mathfrak{g}_{p}\right)=(1+\mu)^{N\left(\mathcal{g}_{p}\right)} \mu^{n-p-N\left(\mathcal{g}_{p}\right)}
$$

where $p(0<p<n)$ is the number of sites in $y_{p}$ and $N\left(g_{p}\right)$ is the number of holes between the sites of $g_{p}$, when taking into account that 1 and $n$ are adjacent. Due to the periodic boundary conditions, $N\left(\mathcal{F}_{p}\right)$ is also equal to the number of connected clusters of sites in $y_{p}$ (Fig. 10). Note that this equality would fail if $p=n$, this cannot happen because $\mathcal{C}$ would be empty.

To prove (B.8b), we have just to recall the fact that

$$
d_{n}=\alpha(\phi)
$$

and to use (B.27). In this case ( $p=0)$, the number of holes is not equal to the number of connected clusters, and we take the convention that $N$ is equal to the number of clusters, that is :

$$
N(\phi)=0 .
$$

Therefore

$$
d_{n}=\alpha(\phi)=(1+\mu)^{N(\phi)} \mu^{n-N(\phi)}+f_{n-1}-f_{n} .
$$

3. Consistency of the factorization hypothesis For any root $\mathcal{C}$, we define $\hat{a}(\mathcal{C})$ by

$$
b(\mathcal{C})=\mu^{m(\mathcal{C})} \hat{a}(\mathcal{C})+f_{n-1}-f_{n} .
$$

We have to prove that $\hat{a}(\mathcal{C})=a(\mathcal{C})$.

(i) First we prove it for the roots $\mathcal{C}_{j}$ defined in relation 1 . One has

$$
b\left(\mathcal{C}_{j}\right)=\sum_{\mathbb{C}^{\prime}}^{\prime} a\left(\mathcal{C}^{\prime}\right) .
$$

Because the root $\mathcal{C}_{j}$ has a single hole of $j$ sites, there are $(j-1)$ consecutive sites which must be empty (are forbidden) in all the configurations $\mathrm{C}^{\prime}$ of the sum $\Sigma^{\prime}$.

One can write $b\left(\mathcal{C}_{j}\right)$ as :

$$
\begin{aligned}
b\left(\mathcal{C}_{j}\right)= & f_{j-1} d_{n-j-1}+2 f_{j} d_{n-j-2}+ \\
& +3 f_{j+1} d_{n-j-3}+\cdots+(n-2-j) f_{n-2} d_{0} \\
& +(n-1-j) f_{n-1} .
\end{aligned}
$$

The first term corresponds to roots where the $(j-1)$ forbidden sites are in a hole of size exactly $j-1$. The 2 nd term corresponds to roots where these $(j-1)$ forbidden sites are in a hole of size exactly $j$. And so on...

This sum gives for $j=n-1$ and $j=n-2$, using (41),

$$
\begin{aligned}
b\left(\mathcal{C}_{n-1}\right) & =f_{n-2}+2 f_{n-1}=\mu f_{n-1}+f_{n-1}-f_{n} \\
b\left(\mathcal{C}_{n-2}\right) & =(1+\mu) f_{n-3}+2 f_{n-2}+3 f_{n-2} \\
& =\mu^{2} f_{n-2}+f_{n-1}-f_{n} .
\end{aligned}
$$

Thus the relation

$$
b\left(\mathcal{C}_{p}\right)=\mu^{n-p} f_{p}+f_{n-1}-f_{n}
$$

is true for $p=n-1$ and $p=n-2$.

By subtracting equation (B.29) for $j$ to equation (B.29) for $j+1$, one gets

$$
\begin{array}{r}
b\left(\mathcal{C}_{j}\right)=b\left(\mathcal{C}_{j+1}\right)+f_{j-1} d_{n-j-1}+f_{j} d_{n-j-2}+\cdots+ \\
+f_{n-2} d_{0}+f_{n-1}
\end{array}
$$

and by another subtraction one finds

$$
\begin{aligned}
b\left(\mathcal{C}_{j}\right)-b\left(\mathcal{C}_{j+1}\right)=b\left(\mathcal{C}_{j+1}\right)-b\left(\mathcal{C}_{j+2}\right) & + \\
& +f_{j-1} d_{n-j-1} .
\end{aligned}
$$

Replacing $d_{n-j-1}$ by its expression (see B.25) and using the relation (41) for the $f_{i}$, one gets that if (B.30) is true for $p \geqslant j+1$, it remains true for $j$. Then, as it is true for $p=n-1$ and $n-2$, it is true for any $p$, $0 \leqslant p \leqslant n-1$.

Therefore, from (B.28), $\hat{a}\left(\mathrm{C}_{p}\right)=f_{p}$, that is

$$
0 \leqslant p \leqslant n-1 \quad \hat{a}\left(\mathcal{C}_{p}\right)=a\left(\mathcal{C}_{p}\right) .
$$


(ii) Let us now take a general configuration $\mathfrak{C}$. From the formula (B.7) shown in part 1, and with (B. $8 a)$ and (B.8b), one has

$$
\begin{array}{r}
b(\mathcal{C})=\sum_{p=0}^{M}(-1)^{p} \sum_{\left\{\not_{p}\right\}} \mu^{n-p}\left(\frac{1+\mu}{\mu}\right)^{N\left(\gamma_{p}\right)}+ \\
+f_{n-1}(\mu)-f_{n}(\mu)
\end{array}
$$

where $\mathfrak{g}_{p}$ is any set of $p$ forbidden sites, and $N\left(\mathcal{F}_{p}\right)$ the number of holes in $\mathcal{J}_{p}$ as explained at the end of part 2. We recall that $I(\mathcal{C})$ is the set of these forbidden sites, which have to be empty in any successor of $\mathrm{C}$. If $\mathcal{C}$ has (exactly) $K$ holes of sizes $j_{1}, j_{2}, \ldots, j_{K}$ (some of them may be of equal sizes), one has

$$
n-m(\mathcal{C})=\sum_{i=1}^{K} j_{i}
$$

$I(\mathcal{C})$ contains $M=\sum_{i=1}^{K}\left(j_{i}-1\right)$ sites and is the union of $K$ non adjacent subsets, say $\left\{I_{i}(\mathrm{C}), i=1, K\right\}$, each $I_{i}(\mathrm{C})$ containing $\left(j_{i}-1\right)$ adjacent sites $\left(I_{i}(\mathcal{C})\right.$ may be $\phi$ if $j_{i}=1$ ). (See Fig. 9.) Then any subset $J_{p}$ of $I(\mathcal{C})$ is the union of $K$ disjoint subsets, say ${ }_{p}{ }_{p i}$, with Card $\dot{y}_{p_{i}}=p_{i}$ and $\dot{y}_{p_{i}} \subset I_{i}(\mathrm{C})$ (one may have $p_{i}=0$, that is $g_{p_{i}}=\phi$ ). Then one has :

$$
\left.\begin{array}{rl}
p & =\sum_{i=1}^{K} p_{i} \\
g_{p} & =\bigcup_{i=1}^{K} j_{p_{i}}
\end{array}\right\} .
$$

We recall that $N\left(\mathfrak{f}_{p}\right)$ is the total number of clusters in $\mathcal{F}_{p}$ (see Fig. 10). This is always true because for any $\mathcal{C}$, Card $I(\mathcal{C})<n$.
Then, as the subsets $\mathfrak{f}_{p_{i}}$ are disjoint, $N\left(\mathfrak{f}_{p}\right)$ is also the sum over $i$ of the numbers of clusters in $\gamma_{p_{i}}$ that is of $N\left(\mathcal{F}_{p_{i}}\right)$. If one $\mathcal{F}_{p_{i}}$ is $\phi$, its contribution to this sum is zero, in agreement with the convention $N(\phi)=0$. Then one has

$$
N\left(\mathfrak{j}_{p}\right)=\sum_{i=1}^{K} N\left(\mathfrak{j}_{p_{i}}\right) .
$$

It is important to note that the convention $N(\phi)=0$ was made such that $\left(\frac{1+\mu}{\mu}\right)^{N\left(\gamma_{p}\right)}$ gives 1 when $p$ is 0 .

Now, from (B.28) and (B.32) one has

$$
\hat{a}(\mathrm{C})=\sum_{p=0}^{M}(-1)^{p} \sum_{\left\{\gamma_{p}\right\}} \mu^{n-m(\mathfrak{C})-p}\left(\frac{1+\mu}{\mu}\right)^{N\left(\gamma_{p}\right)} .
$$

From(B.33) to(B.36) one gets

$$
\begin{aligned}
\hat{a}(\mathcal{C})=\sum_{p=0}^{M} \sum_{\left\{\gamma_{p_{i}} ; 1 \leqslant i \leqslant K\right\}} \times \\
\times\left[\prod_{i=1}^{K}(-1)^{p_{i}} \mu^{j_{i}-p_{i}}\left(\frac{1+\mu}{\mu}\right)^{N\left(\gamma_{p_{i}}\right)}\right]
\end{aligned}
$$

that is

$$
\hat{a}(\mathcal{C})=\prod_{i=1}^{K}\left[\sum_{p_{i}=0}^{j_{i}-1}(-1)^{p_{i}} \mu^{-p_{i}+j_{i}} \sum_{\left\{\partial_{p_{i}}\right\}}\left(\frac{1+\mu}{\mu}\right)^{N\left(\gamma_{p_{i}}\right)}\right] .
$$

For the particular case $K=1$, this expression reduces to $\hat{a}\left(\mathcal{C}_{j_{1}}\right)$ which is equal to $a\left(\mathcal{C}_{j_{1}}\right)$ (see Eq. B.31). Thus for any $\mathrm{C}$ :

$$
\hat{a}(\mathcal{C})=\prod_{i=1}^{K} \hat{a}\left(\mathcal{C}_{j_{i}}\right)=\prod_{i=1}^{K} a\left(\mathcal{C}_{j_{i}}\right)=a(\mathcal{C})
$$

and that completes the proof.

\section{References}

[1] Blease, J., J. Phys. C (Solid State Phys.) 10 (1977) 917.

[2] Oвuknov, S. P., Physica 101A (1980) 145.

[3] Kertesz, J. and Vicsek, T., J. Phys. C 13 (1980) L343.

[4] Dhar, D. and Barma, M., J. Phys. C 14 (1981) L1.

[5] Kinzel, W. and Yeomans, J. M., J. Phys. A (Math. Gen.) 14 (1981) L163.

[6] Domany, E. and Kinzel, W., Phys. Rev. Lett. 47 (1981) 5 ;

Wu, F. Y. and Stanley, H. E., Phys. Rev. Lett. 48 (1982) 775.

[7] Klein, W. and Kinzel, W., J. Phys. A 14 (1981) L405.

[8] Redner, S., Phys. Rev. B 25 (1982) 3242.

[9] DAy, A. R. and Lubensky, T. C., J. Phys. A 15 (1982) L285.

[10] Redner, S. and Yang, Z. R., J. Phys. A 15 (1982) L177.

[11] Dhar, D., Phani, M. K. and Barma, M., J. Phys. A 15 (1982) L279.

[12] Derrida, B. and De Seze, L., J. Physique 43 (1982) 475.

[13] Nightingale, M. P., Physica 83A (1976) 561.
[14] des Cloizeaux, J., J. Physique Colloq. 39 (1978) C2-135.

[15] Guttmann, A. J. and Gaunt, D. S., J. Phys. A 11 (1978) 949.

[16] Klein, D. J., J. Stat. Phys. 23 (1980) 561.

[17] Fisher, M. E. and BARBer, M. N., Phys. Rev. Lett. 28 (1972) 1516.

[18] BRÉZIN, E., private communication.

[19] The shift $(\Delta T)_{n} \sim n^{-1 / v}$ seems to be valid for many models except the spherical model which is very particular.

[20] Daoud, M. and De Gennes, P. G., J. Physique 38 (1977) 85.

[21] Guttmann, A. J. and Wittington, S. G., J. Phys. A 11 (1978) L107.

[22] Feller, W., "An Introduction to Probability Theory and its Applications » (Wiley) 1968, p. 99.

[23] Lubensky, T. C. and Vannimenus, J., J. Physique-Lett. 43 (1982) L-377.

[24] Redner, S. and Coniglio, A., J. Phys. A 15 (1982) L273. 